\title{
Modern Neuroscience and Near-Death Experiences: Expectancies and Implications. Comments on "A Neurobiological Model for Near-Death Experiences"
}

\author{
Michael A. Persinger, Ph.D., C. Psych. \\ Laurentian University, Sudbury, Ontario
}

If correspondences between phenomenological experiences and the intricate activity of the brain continue to be established at the present rate, then the potential for predicting the details and the occurrences of both frequent and anomalous cognitive processes will be inevitable. Juan C. Saavedra-Aguilar and Juan S. Gómez-Jeria's "A neurobiological model for near-death experiences" demonstrates that even a cursory understanding of neural pathways and brain metabolism can predict the general characteristics of experiences that have been traditionally considered beyond the limits of a neurophilosophy. It has become clear that many universal experiences of the human species originate from the marked similarities of its approximately 5 billion brains.

The authors' basic model for near-death experiences (NDEs) is a specialized variant of an approach that implicitly emphasizes a neural cause to all phenomenology (Persinger, 1983, 1987). NDEs are assumed to be generated by stress-induced hypoxia and ischemia, most likely vasospasm, that preferentially affect limbic structures, such as the amygdala and hippocampus, and the paralimbic orbital frontal,

Dr. Persinger is Professor of Neuroscience and Psychology, and Coordinator of the Behavioral Neuroscience Program, at Laurentian University. Requests for reprints should be addressed to him at the Department of Psychology, Laurentian University, Ramsey Lake Road, Sudbury, Ontario, P3E 2C6, Canada. 
temporal, insular and cingulate cortices. Once they are stimulated, the correlative functions dominate the phenomenological continuum. Whereas the serial emergence of the successive, general themes of the NDE reflect progressive involvement of limbic areas as the chemical consequences of the initiating stimulus spreads, the specific details such as the type of mystical entity, are supplied by the person's behavioral experience and expectancies. Other individual differences in experiential detail are presumed to be a consequence of the idiosyncratic neuronal connections at the microlevel.

From the perspective of a modern neuroscientist, both the neuroanatomy and neurochemistry described by the authors are adequate, given the limitations of generalizing from non-human to human brain, and contemporary. A vast clinical and surgical literature (Stevens, 1982) indicates that floating and rising sensations, out-of-body-experiences, personally profound mystical and religious encounters, visual and auditory experiences, and dream-like sequences are evoked, usually as single events, by electrical stimulation of deep, mesiobasal temporal lobe structures. The potent role of neuropeptides, especially the endogenous opiates and the stress hormones corticotrophic releasing factor (CRF) and adrenal corticotrophic hormone (ACTH), in the control of electrical lability of the hippocampus and amygdala is clear.

There is little doubt that the class of experiences that comprise mystical experiences in general, and NDEs in particular, is strongly correlated with temporal lobe activity. Indeed there are multivariate contributions to its functional level, including specific drugs; anomalous vasospasm, a major mechanism by which hallucinogenic effects are generated (Altura \& Altura, 1981); grief-related stress; meditational techniques; and even certain exercise schedules. Kate Makarec and I have found that all of the major components of the NDE, including out-of-body experiences, floating, being pulled towards a light, hearing strange music, and profound meaningful experiences can occur in experimental settings during minimal electrical current induction within the temporal region due to exogenous spike-and-wave magnetic field sources. However, these induced experiences are fragmented and variable, whereas in NDEs these sensations are integrated and focused within a brief period.

The assumptions of the Saavedra-Aguilar and Gómez-Jeria model are strongly rooted in known neurofunction. Two examples can be given. The odd noises that are frequently heard during the early stages of NDEs may indeed reflect the unique vasculature and the hypoxic sensitivity of the cochlear region; one of the initial responses of neurons within a marginally hypoxic region is to display nonspecific com- 
pensatory firing, a consequence of which would be the psychological experience of unfamiliar sounds. The occurrence of the life review would be commensurate with the model that the hippocampus acts as an "index system" (Teyler \& DiScenna, 1984), while memory equivalents are stored in the 200 million columns that comprise the cerebral cortices. Generalized or nonspecific epileptiform activity in the hippocampus could activate large numbers of cortical columns (and memories) that are typically temporally and contextually separate.

Like all models, this one becomes complicated when quantitative estimates are attempted. How much ischemia or hypoxia is required to induce the NDE? Stated alternatively, why don't more people display NDEs within surgical, accident, and cardiovascular crises? The explanation of postepisodic amnesia is real, but within a theoretical context becomes a weak, default option. No doubt there are many factors that might predispose a person to reporting a NDE. Obvious factors include cognitive style or personality, both of which are intimately involved with temporal lobe function due to the confounding effects of language and the importance of affect in experience; a history of subclinical complex partial epileptic signs, that normally increase with age; recondite, anomalous vascular distribution within the hippocampus, that is particularly prone to transient vasospasm because of the small arterial caliber and remarkable geometric variability; or increased sensitivity of ACTH receptors as a result of psychological distress.

The fact that fatigue, alcohol consumption, or surgically-induced pharmacological conditions existed on the days of NDEs strongly suggests that they might be triggered during the compensatory overshoot in neural activity. For example, alcohol inhibits N-methyl-D-aspartate (NMDA) receptor activity (Lovinger, White, and Weight, 1989). Consequent increases in NMDA stimulation and the correlative calcium influx are sufficient to generate enhanced local depolarization, the first step in generalized paroxysmal discharge. Such factors are important to accommodate if the NDE model is to be supported by prediction.

The degree to which anxiety, such as anxiety over real or imagined impending death, acts as a predisposing factor to the NDE is important to determine. Recent positron emission tomography (PET) experiments (Reiman, Fusselman, Fox, and Raichle, 1989) with normal subjects indicated that this aversive experience is associated with specific increases in blood flow within the parahippocampal region, the insula, and the adjacent claustrum. These same areas, if stimulated excessively, would generate anomalous memory-like experiences, sensations of body images, and visual sensations that involve glows around perceptual boundaries. 
Considering the spatial-temporal similarity in brain activity, many of the early stages of NDEs should be similar to those of panic attacks, which are often evoked by excess lactate production, a common correlate of cerebral ischemia. Panic attacks can involve sensations of fear or anxiety, out-of-body experiences (OBEs), depersonalization, feelings of a presence, and alterations in sensory information. PET data have suggested that differential blood flow occurs within the parahippocampal regions of the two hemispheres, a key area of the present NDE hypotheses. Although the relative absence of positive affect in panic attacks compared to NDEs could be rationalized by assuming that NDEs occur with more severe impairments of consciousness, the differences must be reconciled according to neuromechanism.

Accommodation of four aspects of NDEs could facilitate the usefulness of the model. First, the neurolevel at which the model operates approaches that from which personality and consciousness emerge. Further elaboration of the personality factors that promote the occurrence and certainly the recall of the NDE would be useful. In my clinical experience, the same people who have limbic-induced cardiovascular failure, due to enhanced temporal lobe sensitivity and specific cognitive styles, are those who are prone to NDEs. If the model is correct, then people who have more nonpathological temporal lobe signs should have more frequent incidence of experiences that comprise the NDE, such as OBEs and mystical experiences. There is clear empirical support for this supposition (Pesinger and Makarec, 1987).

Second, even with the release of opiates within delta receptor regions of the amygdala, the hierarchical organization of this structure should not be altered. Because a significant area of this region is involved with aversive experiences, the model should predict that a small portion (1\% to $5 \%$ ) of NDEs should involve primarily negative affect and images. References to pain, hell, and nightmarish images, often culture specific, must occur.

Third, a direct translation of the pattern of neural firing within a specific cerebral region into the fabric of the NDE would be conclusive. In less exotic settings, the degree of hypermetabolic activity within the left prefrontal region and adjacent caudate is specifically related to the severity of obsessive-compulsive behaviors. Listening to music without lyrics generates more activity in the right non-dominant temporal lobe compared to the left. A direct connection between the vestibulovisual sensation of "the self' being pulled towards a light" and a specific discharge pathway in the limbic region would be conclusive.

Fourth, the concept of generalized depolarization of electrical processes induced by the hypoxia-ischemia of the trauma is too vague. If 
the depolarization were generalized, then NDEs should also contain references to aggression, sexual ideation, and intense fear; the consistent pattern of NDEs suggests that some relatively fixed discharge patterns must occur. Although there is no doubt that the major pathways of microseizure discharge, many of which are not evident with routine electroencephalographic measurements, must pass through structural "gateways" (Gale, 1988) that limit their propagation, the NDE demands involvement of relatively fixed and hierarchical neuronal pathways. The possibility that these pathways might be opened by critical, trauma-related chemical environments in a statedependent manner is worthy of investigation.

There are some major features of the Saavedra-Aguilar and GómezJeria NDE model that have important clinical implications. If NDEs are associated with epileptiform activity, are there longterm sequelae? In one clinical series, approximately $30 \%$ of all patients who recovered from cardiopulmonary resuscitation developed a special form of limbic epilepsy. Because behavioral changes often precede overt signs of this disorder by months or even years, there should be substantial personality changes in NDErs (excluding those complicated by terminal disorders), and NDErs should be more prone to the development of focal or complex partial epilepsy. The type of personality change should be specific to the "temporal lobe pattern" that is seen clinically; symptoms include increasing circumstantiality, religiosity, philosophical pursuits, widening of affect, and a sense of the personal (Bear \& Fedio, 1977).

Ethical responsibility demands that any hypothesis that might evoke psychological distress in another human being should be considered carefully; the extreme interpretation of NDEs is that they are illusionary. However, I do not perceive a conflict with those researchers who believe that NDEs are real rather than artifacts of the brain's construction. Even the most conservative neuroscientist should agree with Vernon Mountcastle's statement: "sensation is an abstraction, not a replication, of the real world" (Mountcastle, 1975, p. 109). This abstraction is coupled to the microstructural organization of the brain that is determined by both genetics and experience.

The relation between neuronal microstructure and the perception of reality is important because they are not fixed in phenomenological time. It is essential to realize that neuroplasticity can be promoted by stimulation of NMDA receptor activity, a component of the authors' model; reorganization of neural pathways does occur within the brains of mammals, including humans, who have histories of focal electrical activity (Sutula, Cascino, Cavazos, Parada, and Ramirez, 1988). If 
indeed structure dictates function, then the type of microstructural changes correlated with the NDE could forever alter the NDErs' detection of what comprises reality. Even the transient changes that occur during an NDE might allow the brief detection of information that has been traditionally considered as parapsychological.

The final logical implication that a neurobiological model of NDEs evokes is their survival value. Although there are instances of counterproductive and even fatal genetic predispositions, most behaviors have adaptive value for the species. The proposed neuroanatomical region, especially the anterior cingulate and deep temporal lobe structures, from which NDEs are presumed to emerge are intimately involved with species-specific behaviors, bonding between members of a group, and parental care. These same regions are actively integrated into the sense of self and its perspective within time and space. These areas show marked dysfunction when the boundaries of the self are distorted, as in schizophrenia, or are eliminated, as in suicide.

One might speculate that as the protoconsciousness of the human brain emerged during those early periods when the hippocampal formation underwent massive restructuring (Stephen, 1983) and change in relative position, the uninhibited fear of dissolution or death of the self would have altered its emergence. Mystical and religious experiences in general and the NDE in particular can be seen as adaptive responses that emerged to maintain the fragile phenomenon of the human sense of self.

\section{References}

Altura, B. T. and Altura, B. M. (1981). Phencyclidine, lysergic acid diethylamide and mescaline: Cerebral artery spasms and hallucinogenic activity. Science, 212, 1051-1052.

Bear, D. and Fedio, P. (1977). Quantitative analysis of interictal behavior in temporal lobe epilepsy. Archives of Neurology, 34, 454-467.

Gale, K. (1988). Progression and generalization of seizure discharge: Anatomical and neurochemical substrates. Epilepsia, 29 (Suppl. 2), 15-34.

Lovinger, D. M., White, G., and Weight, F. (1989). Ethanol inhibits NMDA-activated ion current in hippocampal neurons. Science, 243, 1721-1723.

Mountcastle, V. B. (1975). The view from within: Pathways to the study of perception. Johns Hopkins Medical Journal, 136, 109-131.

Persinger, M. A. (1987). Neuropsychological bases of god beliefs. New York, NY: Praeger.

Persinger, M. A. (1983). Religious and mystical experiences as artifacts of temporal lobe functions: A general hypothesis. Perceptual and Motor Skills, 57, 1255-1262.

Persinger, M. A., and Makarec, K. (1987). Temporal lobe epileptic signs and correlative behaviors displayed by normal populations. Journal of General Psychology, 114, 179-195. 
Reiman, E. M., Fusselman, M. J., Fox, P. T., and Raichle, M. E. (1989). Neuroanatomical correlates of anticipatory anxiety. Science, 243, 1071-1074.

Stephan, H. (1983). Evolutionary trends in limbic structures. Neuroscience and Biobehavioral Reviews, 7, 367-374.

Stevens, J. R. (1982). Sleep is for seizures: A new interpretation of the role of phasic events in sleep and wakefulness. Pp. 249-264 in M. B. Sternman, M. N. Shouse, \& P. Passouant (eds.), Sleep and epilepsy. New York, NY: Academic Press.

Sutula, T., Cascino, G., Cavazos, J., Parada, I., and Ramirez, L. (1988). Hippocampal synaptic reorganization in partial complex epilepsy: Evidence for mossy fiber sprouting in epileptic human temporal lobe. Annals of Neurology, 24, 134-135.

Teyler, T. K. and DiScenna, P. (1984). The topological anatomy of the hippocampus: A clue to its function. Brain Research Bulletin, 12, 711-719. 